\title{
Highlights from the Pierre Auger Observatory
}

\author{
Antoine Letessier-Selvon • \\ for the Pierre Auger Collaboration
}

Received: 28 April 2014 / Published online: 18 June 2014

(C) Sociedade Brasileira de Física 2014

\begin{abstract}
The Pierre Auger Observatory is the world's largest cosmic ray observatory. Our current exposure reaches nearly $40,000 \mathrm{~km}^{2} \mathrm{sr}$ and provides us with an unprecedented quality data set. The performance and stability of the detectors and their enhancements are described. Data analyses have led to a number of major breakthroughs. Among these, we discuss the energy spectrum and the searches for large-scale anisotropies. We present analyses of our $X_{\max }$ data and show how it can be interpreted in terms of mass composition. We also describe some new analyses that extract mass-sensitive parameters from the $100 \%$ duty cycle surface detector (SD) data. A coherent interpretation of all these recent results opens new directions. The consequences regarding the cosmic ray composition and the properties of ultrahigh-energy cosmic ray (UHECR) sources are briefly discussed.
\end{abstract}

Keywords Pierre Auger Observatory · Highlights ·

Ultrahigh-energy cosmic rays

\section{The Pierre Auger Observatory}

The Pierre Auger Collaboration is composed of more than 500 members from 19 different countries. The world's largest observatory [1] is located in the southern part of the province of Mendoza in Argentina. It is dedicated to the

Full author list: http://www.auger.org/archive/authors201305.html (for the Pierre Auger Collaboration)

A. Letessier-Selvon $(\triangle)$

Laboratoire de Physique Nucléaire et des Hautes Énergies,

Université Pierre et Marie Curie et Université Denis Diderot, CNRS/IN2P3, Paris, France

e-mail: antoine.letessier-selvon@in2p3.fr studies of ultrahigh-energy cosmic ray (UHECR) from an energy of a fraction of an Exa electron-Volts $(\mathrm{EeV})^{1}$ to the highest energy ever observed at several hundreds of EeV. The observatory comprises several instruments working in symbiosis:

- A surface detector (SD) array of 1,600 water Cherenkov detectors (WCDs) arranged on a regular triangular grid of $1,500 \mathrm{~m}$ and covering $3,000 \mathrm{~km}^{2}$ [2].

- Four sites with fluorescence detector (FD) (each site contains six telescopes for a total of $180^{\circ}$ azimuth by $30^{\circ}$ zenith field of view) [3].

- A subarray, the infill, with 71 water Cherenkov detectors on a denser grid of $750 \mathrm{~m}$ covering nearly $30 \mathrm{~km}^{2}$ [4]. This subarray is part of the AMIGA extension that will also have buried muon counters at each 71 WCD locations (seven are in place [30]).

- Three high-elevation Auger telescopes (HEATs) located at one of the fluorescence sites [5] dedicated to the fluorescence observation of lower-energy showers.

- A subarray of 124 radio sensors (Auger Engineering Radio Array (AERA)) working in the $\mathrm{MHz}$ range and covering $6 \mathrm{~km}^{2}$ [6].

- A subarray of 61 radio sensors (Extensive Air Shower Identification with Electron Radiometer (EASIER)) working in the $\mathrm{GHz}$ range and covering $100 \mathrm{~km}^{2}$ [7].

- Two GHz imaging radio telescopes, AMBER [8] and MIDAS [9], with $14^{\circ} \times 14^{\circ}$ and $10^{\circ} \times 20^{\circ}$ field of views, respectively.

The last three items are R\&D on the detection of extensive air showers using the radio emission of the EM cascade in the atmosphere.

$\overline{{ }^{1} 1 \mathrm{EeV}=10^{18}} \mathrm{eV}$ or $0.16 \mathrm{~J}$ 
In total, the Auger Collaboration has provided to this conference 32 contributions [10-43], including three contributions [41-43] done in collaboration with the Telescope Array (TA) collaboration [44]. These contributions describe a wide range of detector techniques, analysis tools, monitoring system, and scientific results developed and produced by the collaboration. In this short highlight, only a fraction of those contributions can be presented.

After a brief description of the detector status and data selection, we present the updated energy scale and corresponding energy spectra, as measured by various components of the observatory. We also report on the measurements of the first two moments (mean and variance) of the longitudinal shower profile $X_{\max }$ distributions in several energy bins and interpret them in terms of mass composition using the recent update of the high-energy generators $[50,51]$.

We describe new analysis techniques that allow us to measure the muonic content of extensive air showers. The analyses, based on the SD data set, profit from the high statistics from this detector with nearly $100 \%$ duty cycle. They allow us to confront models for hadronic interactions at high energies with data at the highest energy and also to recover mass-sensitive parameters independently from FD measurements.

Last but not least, we report on the searches for largescale anisotropies in the $\mathrm{EeV}$ range and their consequences.

\subsection{Status}

The hybrid concept has been pioneered by the Auger Collaboration and allows, among other things, for calibration of the SD that is fully data driven, thus avoiding the uncertainties related to the use of Monte Carlo-simulated showers. Such calibration allows the transfer of the high-precision calorimetric information collected by the FD to the $100 \%$ duty cycle SD. In the following, the term hybrid will also refer to those events that are observed simultaneously by $\mathrm{SD}$ and FD, which form a specific data set called the hybrid data.

To fully benefit from this technique, it is, however, mandatory to monitor with extreme precision both the detector activity and the atmospheric experimental conditions. Out of the major correction terms applied to the FD energy, the atmospheric transmission through aerosols has the largest time variation and must be followed most closely.

The Auger site is equipped with an extensive set of instruments that measure the atmospheric conditions [35-37]. These instruments allow us to determine, within accuracies of a few percent, the hourly vertical aerosol optical depth (VAOD) as well as to obtain a sky representation of the cloud coverage.

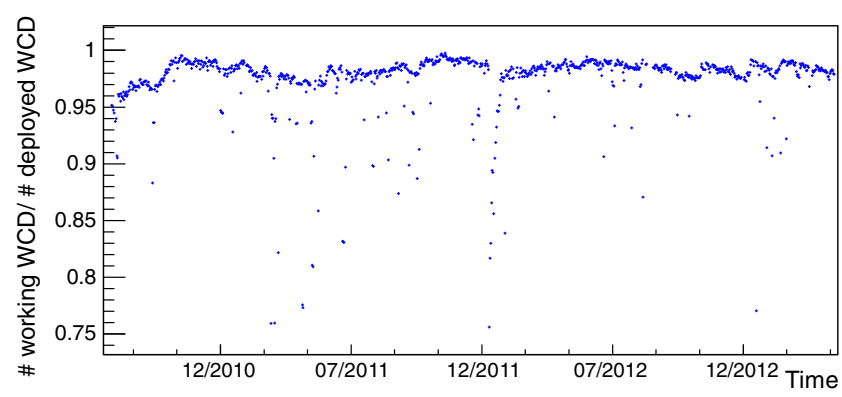

Fig. 1 Normalized number of active SD stations as a function of time [14]

In addition to the atmosphere monitoring, an extensive collection of hardware and software tools has been developed, and these are used to monitor (up to second by second) the activity of different detector components. This provides online and long-term detector as well as data quality control [14]. Examples of such monitoring information are shown in Figs. 1 and 2.

In Fig. 1, the activity of each individual WCD station is reported (the data averaged in the plot were collected each second). One can visually measure the nearly constant and efficient activity of the array which is about $98 \%$ on average.

In Fig. 2, we show the hybrid on-time fraction of our FD sites. Such monitoring allows for a precise determination of the experimental exposure as well as for a precise control of the data quality.

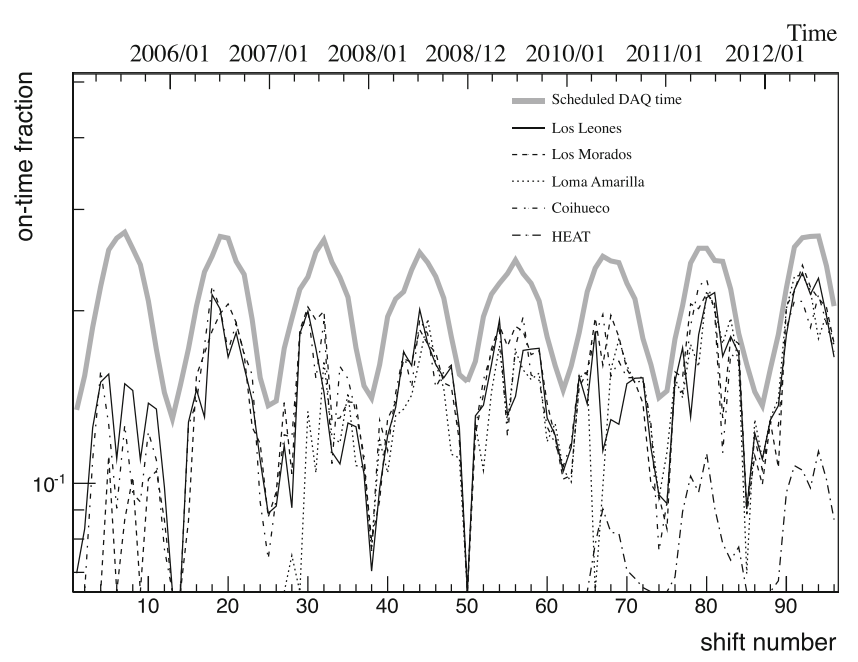

Fig. 2 Hybrid on-time fraction for the four FD sites and HEAT. The thick gray line defines the scheduled data taking (limited to nights with less than $60 \%$ moon fraction) [14] 
Table 1 Summary of the experimental parameters describing data of the different measurements at the Pierre Auger Observatory

\begin{tabular}{|c|c|c|c|c|}
\hline & $\begin{array}{l}\text { Auger SD } \\
1,500 \mathrm{~m} \text { vertical }\end{array}$ & $1,500 \mathrm{~m}$ inclined & $750 \mathrm{~m}$ vertical & Auger hybrid \\
\hline Data taking period & $\begin{array}{l}\text { January } 2004- \\
\text { December } 2012\end{array}$ & $\begin{array}{l}\text { January } 2004- \\
\text { December } 2012\end{array}$ & $\begin{array}{l}\text { August 2008- } \\
\text { December } 2012\end{array}$ & $\begin{array}{c}\text { November 2005- } \\
\text { December } 2012\end{array}$ \\
\hline Exposure $\left(\mathrm{km}^{2} / \mathrm{sr} /\right.$ year $)$ & $31,645 \pm 950$ & $8,027 \pm 240$ & $79 \pm 4$ & - \\
\hline Zenith angles $\left({ }^{\circ}\right)$ & $0-60$ & $62-80$ & $0-55$ & $0-60$ \\
\hline Threshold energy, $E_{\text {eff }}(\mathrm{eV})$ & $3 \times 10^{18}$ & $4 \times 10^{18}$ & $3 \times 10^{17}$ & $10^{18}$ \\
\hline No. of events $\left(E>E_{\mathrm{eff}}\right)$ & 82,318 & 11,074 & 29,585 & 11,155 \\
\hline No. of events (golden hybrids) & 1,475 & 175 & 414 & - \\
\hline Energy calibration $(\mathrm{A})(\mathrm{EeV})$ & $0.190 \pm 0.005$ & $5.61 \pm 0.1$ & $(1.21 \pm 0.07) \times 10^{-2}$ & - \\
\hline Energy calibration (B) & $1.025 \pm 0.007$ & $0.985 \pm 0.02$ & $1.03 \pm 0.02$ & - \\
\hline
\end{tabular}

Numbers of events are given above the energies corresponding to full trigger efficiency [16]

\subsection{Data Sets}

The data sets used for the various analyses presented here and at the conference have minor variations from one analysis to the next as described in detail in the corresponding conference contributions [10]. However, they share some common features.

The data-taking period extends from 1 January 2004 to 31 December 2012, thus updating the measurements we have published earlier. To ensure an appropriate and accurate reconstruction of the cosmic ray parameters such as the arrival direction and energy or of the characteristics of the shower longitudinal development (e.g., $X_{\max }$ ), several quality cuts are applied. For SD analyses, it is, for example, required that the WCD with the largest signal be surrounded by six working and active WCDs at the time of the event.

Different attenuation characteristics of the electromagnetic and muonic shower components lead to different reconstruction methods for the different associated zenith angle ranges. We distinguish, in particular, between vertical events with a zenith angle $\theta$ between $0^{\circ}$ and $60^{\circ}$ (or

Table 2 Changes to the shower energy at $10^{18} \mathrm{eV}[11]$

\begin{tabular}{lc}
\hline Changes in FD energies at $10^{18} \mathrm{eV}$ & Percent \\
\hline Absolute fluorescence yield & -8.2 \\
New optical efficiency & 4.3 \\
Calibration database update & 3.5 \\
Subtotal (FD calibration) & 7.8 \\
Likelihood fit of the profile & 2.2 \\
Folding with the point spread function & 9.4 \\
Subtotal (FD profile reconstruction) & 11.6 \\
New invisible energy & 4.4 \\
Total & 15.6 \\
\hline
\end{tabular}

$\theta<55^{\circ}$ for the infill) and inclined events with a zenith angle between $62^{\circ}$ and $80^{\circ}$.

As mentioned, the energies of SD events are determined from the cross calibration with the FD using the hybrid data set. The SD size parameters $(S)\left(S_{38}, S_{35}\right.$, and $\left.N_{19}\right)$, for the regular array, $750 \mathrm{~m}$ infill, and inclined data sets, respectively, are related to the FD energy using a calibration curve of the form $E_{F D}=A S^{B}$. The value of those parameters is reported in Table 1 together with the corresponding data set sizes and main characteristics.

The overall uptime and efficiency of the SD is about $98 \%$, while that of the FD is $13 \%$. The energy resolution of the SD alone is $12 \%$ (statistical) above $10 \mathrm{EeV}$, while the angular resolution is less than $1^{\circ}$ in that energy range.

The total exposure, corresponding to the data sets presented in Table 1, is about $40,000 \mathrm{~km}^{2} / \mathrm{sr} /$ year. From now

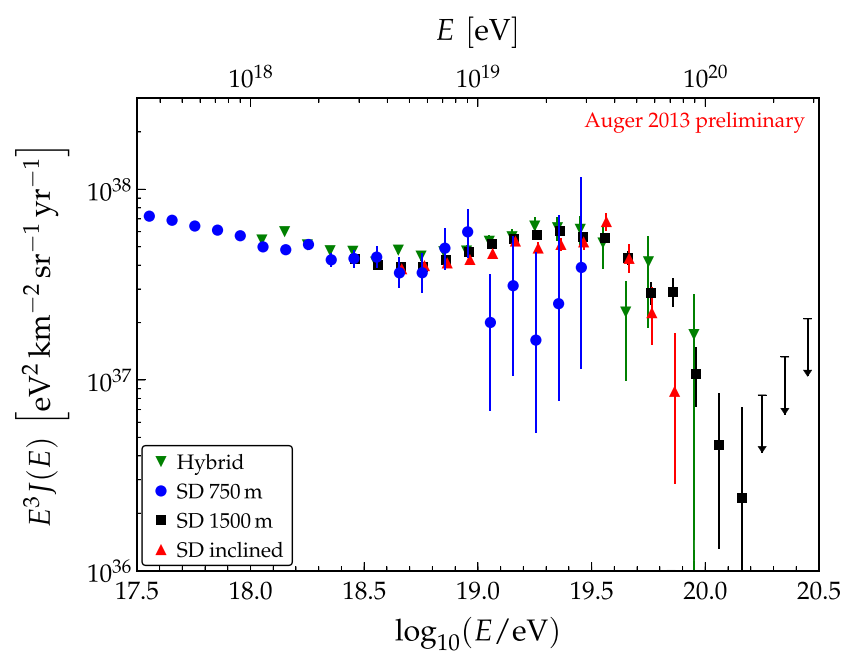

Fig. 3 The Auger energy spectra obtained from various SD and hybrid data sets [16] 


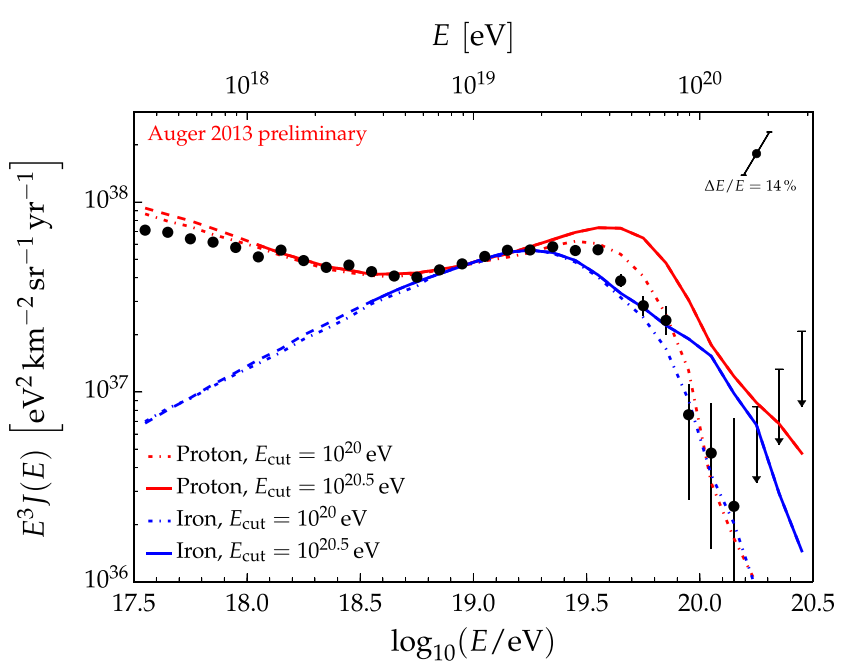

Fig. 4 The combined Auger energy spectrum compared to spectra from different astrophysical scenarios

on, over $6,000 \mathrm{~km}^{2} / \mathrm{sr} /$ year is expected to be collected each year.

It is interesting to note that the combination of our horizontal and vertical data sets gives us a remarkably large sky coverage (up to nearly $50^{\circ}$ declination north). In addition, a recent upgrade of our triggering system, especially at the local WCD level, is being commissioned. It will allow us to bring the energy at which the SD reaches full trigger efficiency from $3 \mathrm{EeV}$ down to about $1 \mathrm{EeV}$ and to significantly improve our photon sensitivities in the $\mathrm{EeV}$ range.

\subsection{Absolute Energy Scale}

On top of the extensive monitoring of the atmosphere and FD operation as a function of time, one must also perform very detailed studies of the light collection efficiencies and frequently calibrate or check the calibration of the instruments. An extensive campaign of measurements and control has been performed at Auger to improve the knowledge of our energy scale and to reduce the systematic uncertainties associated with it [11].

Corrections to the absolute energy scale come from various sources. Among these are the fluorescence yield [46], point spread function measurements performed with our flying light source (the Octocopter now also jointly used at TA [41]), changes in the reconstruction of the shower longitudinal profile, better understanding of the telescope point spread function and accurate simulation of the optics through detailed ray tracing [13], and improvements in the analyses and, in particular, in the estimation of the missing energy [12]. A summary of the changes at a reference energy of $1 \mathrm{EeV}$ is given in Table 2, amounting to $+15.6 \%$. There is a small energy dependence associated with some of those corrections, and the global shift becomes $+11.3 \%$ at $10 \mathrm{EeV}$.

These extensive studies also have allowed better control of the uncertainties associated with each of those corrections. While our overall systematic uncertainty was $22 \%$ at the 32nd ICRC in Beijing, China (in 2011), it is now reduced to $14 \%$.

\section{Spectrum}

After energy calibration, the exposure for each data set (hybrid, infill, SD vertical, and SD horizontal) is carefully evaluated on the basis of our precise monitoring systems. The corresponding spectra are shown in Fig. 3.

Those spectra are combined to form the Auger spectrum as shown in Fig. 4. The combination process relies upon a maximum likelihood method that allows for a normalization adjustment between the various spectra [16]. The corrections, which are well within the normalization uncertainty of the individual spectra, amount to $-6,+2,-1$, and $+4 \%$, respectively. The total number of events comprising the spectrum shown in Fig. 4 is about 130,000 .

This unprecedented statistical accuracy allows a clear identification of two features in the energy spectrum: the ankle and the cutoff at the highest energy. At the ankle, the spectral index changes from $-3.23 \pm 0.07$ to $-2.63 \pm 0.04$ at a break point energy of $5 \mathrm{EeV}$. Above $20 \mathrm{EeV}$, the spectrum starts to deviate from a simple power law, and a flux suppression (a cutoff) is observed. At $E_{50} \%=40 \mathrm{EeV}$, the observed spectrum is half of what is expected from
Fig. 5 Evolution of $\left\langle X_{\max }\right\rangle$ and $\sigma_{X_{\max }}$ as a function of energy. Measurements are from the hybrid data set of Auger [19]
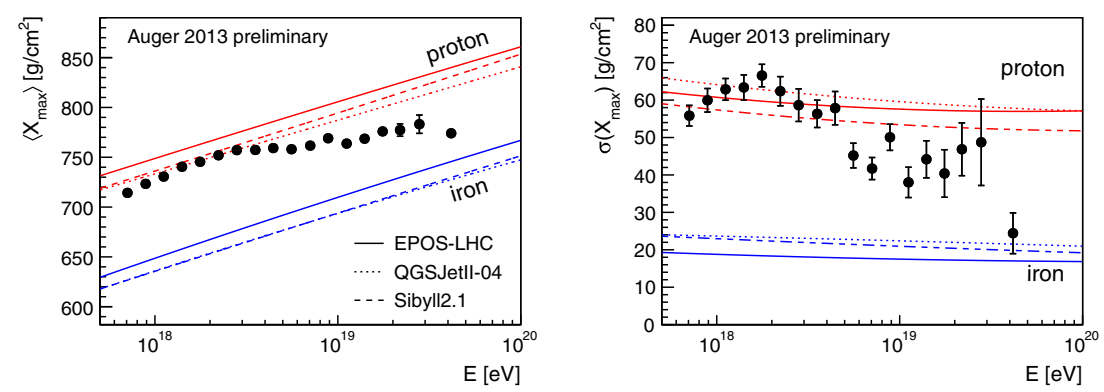

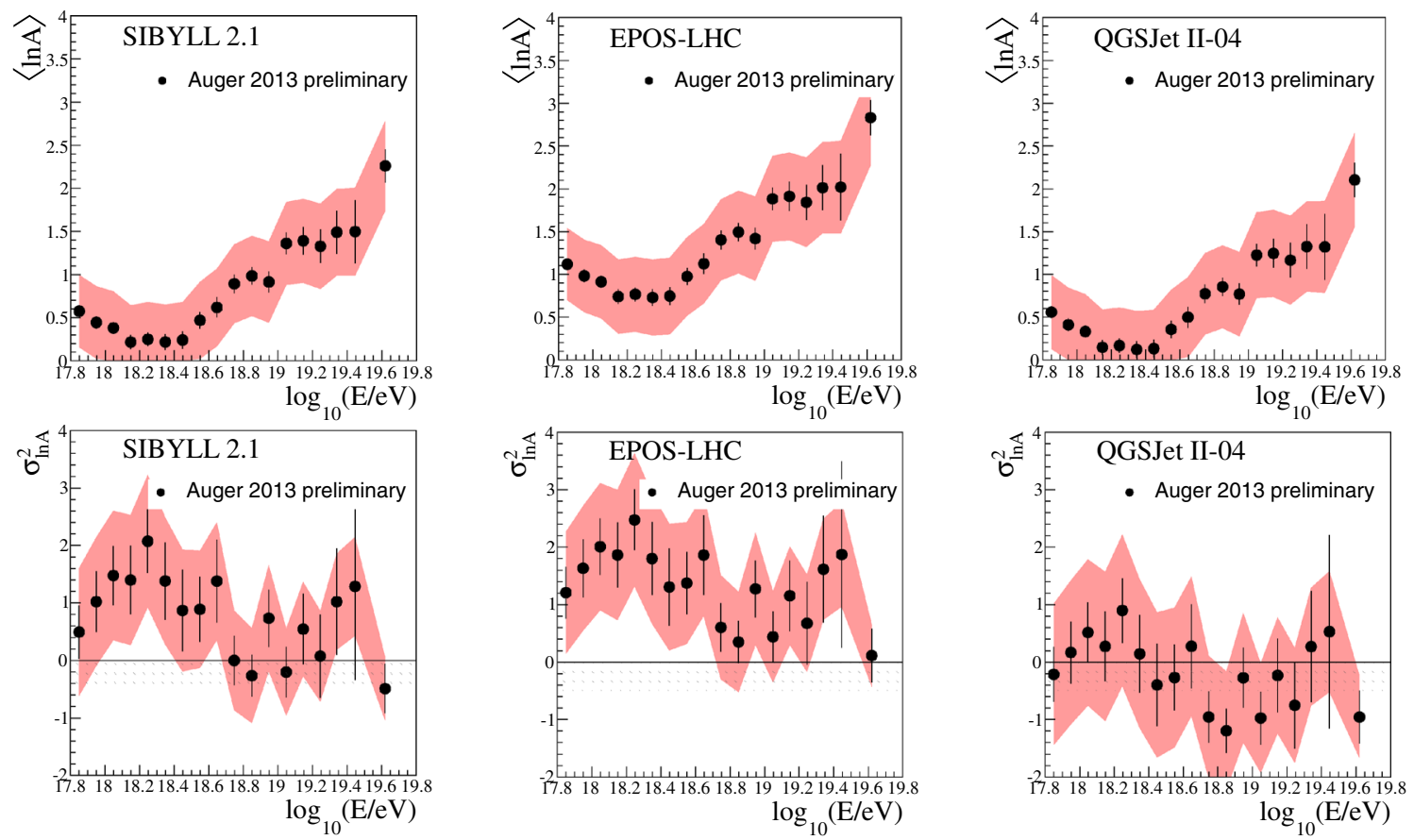

Fig. 6 Conversion to $\langle\ln \mathrm{A}\rangle$ and $\sigma^{2}{ }_{\ln \mathrm{A}}$ using various hadronic interaction models. The red bands indicate the systematic uncertainties [19]

the extrapolation of the power law observed just above the ankle. When compared to a simple continuation of a power law, the significance of the cutoff is more than 20 sigma; however, its origin as that of the ankle is yet to be determined.

These features can originate from interactions of the cosmic rays with the intergalactic radiation field (mainly the cosmic microwave background (CMB)) during their transport from their sources to the Earth. This is the case, for

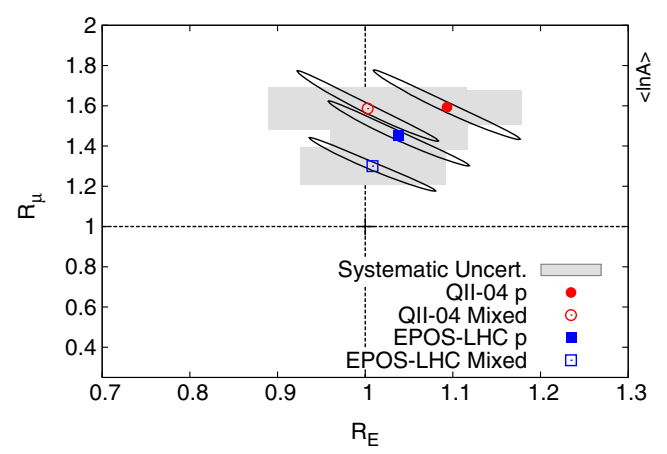

Fig. 7 Value of the energy rescaling parameter $R_{E}$ and muon rescaling parameter $R_{\mu}$ that best represent the Auger hybrid data at $10 \mathrm{EeV}$. The predicted energy is compatible with the observed one ( $R_{E}$ is compatible with 1 within the systematics on the absolute energy scale), while the muon rescaling parameters demand an increase of at least $20 \%$ of the muon size from the models [23] example, of the $\mathrm{e}^{+} \mathrm{e}^{-}$pair or pion production (GreisenZatsepin-Kuzmin (GZK)) from protons of CMB photons for the ankle and the cutoff, respectively, or of the photodisintegration of nuclei. Such features also can originate from the source spatial distributions and/or their acceleration characteristics; in this case, the ankle could sign the transition from a galactic dominated cosmic ray sky to an extragalactic dominated one, while the cutoff would directly reflect the maximum energy reachable by the sources themselves. Various scenarios have been put forward, combining these possible origins in various ways (see, e.g., [45] for an overview).

The models shown in Fig. 4 assume either a pure proton or pure iron composition. The fluxes result from different assumptions of the spectral index $\beta$ of the source injection spectrum and the source cosmological evolution parameter $m$. The maximum energy of the source was set in these particular examples to 100 and $300 \mathrm{EeV}$, the former describing better the data in the cutoff region. The model lines have been calculated using CRPropa [47] and have been validated with SimProp [48].

Despite its high statistical accuracy, the energy spectrum alone is not sufficient to distinguish between the various scenarios. There are simply too many unknowns (source distributions and evolution, acceleration characteristics, cosmic ray mass composition). Other observables such as anisotropies and mass composition parameters will have to be combined to disentangle the situation. 
Fig. 8 Conversion of the $X_{\max }$ and $X_{\max }^{\mu}$ observable to $\langle\ln \mathrm{A}\rangle$ using two different hadronic interaction models EPOS-LHC (left) and QGSJetII-04 (right). While QGSJetIII-04 presents a more coherent conversion, EPOS-LHC offers a better description of the rapidity gap distribution of p-p collision at the LHC. The modification of this distribution in EPOS to better reproduce the LHC p-p data is believed to be responsible for the shift in $X_{\max }^{\mu}$ [53]
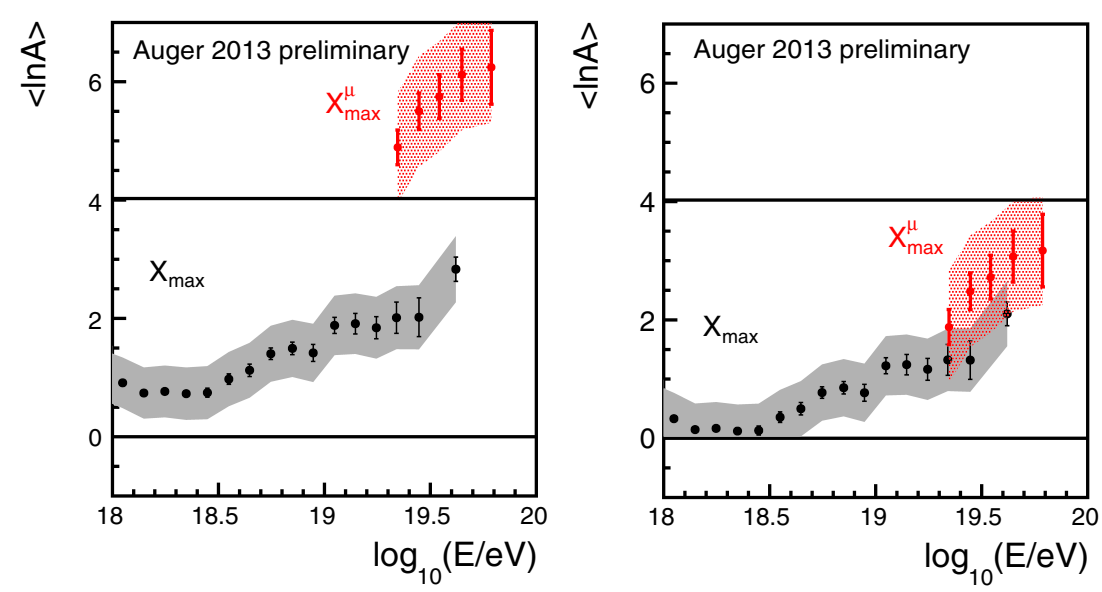

\section{Mass Composition}

The hybrid nature of the Auger Observatory allows for a very precise measurement of the shower longitudinal profile on a subset of less than $10 \%$ of the events (the hybrid data set). The combination of FD and SD allows for a precise determination of the shower geometry which, in turn, allows the measurement of the position of the maximum shower size $\left(X_{\max }\right)$ with an accuracy of better than $20 \mathrm{~g} / \mathrm{cm}^{2}$.

The updated (but preliminary) results regarding the evolution with energy of the first two moments of the $X_{\max }$ distributions are shown in Fig. 5. When compared to the model lines, the data clearly indicate a change of behavior at a few $\mathrm{EeV}$, i.e., in the ankle region.

While predictions of different models may not be an accurate representation of nature for the absolute values of $\left\langle X_{\max }\right\rangle$, hence making it difficult to convert with confidence these data into mass values, they have similar predictions (within $20 \mathrm{~g} / \mathrm{cm}^{2}$ for $\left\langle X_{\max }\right\rangle$ and $10 \mathrm{~g} / \mathrm{cm}^{2}$ for $\sigma_{X_{\max }}$ ) for those parameters. In particular, all models predict that for a constant composition, the elongation rate (slope of the $\left\langle X_{\max }\right\rangle$ evolution) and $\sigma_{X_{\max }}$ are also constant as a function of energy. This is at clear variance from the measurements themselves. Hence, under the hypothesis that no new interaction phenomena in the air shower development come into play in that energy range, the data clearly support that the composition evolves in the ankle region.

While subject to the belief that current interaction models do represent reality, it is possible to convert the measured data into the first two moments of the $\ln \mathrm{A}$ distribution at the top of the atmosphere [52]. This is shown in Fig. 6 using several hadronic interaction models [49-51]. From this conversion, it is possible to interpret the aforementioned evolution as a change from light to medium light composition with a minimum in the average $\ln \mathrm{A}$ just before the ankle, i.e., between 2 and $3 \mathrm{EeV}$. Looking at the $\sigma^{2}{ }_{\operatorname{lnA}}$ plot, one can also argue that the evolution is slow in terms of masses $\left(\sigma^{2} \ln\right.$ A stays below 2 in the whole range, indicating that the mix is between nearby masses rather than between proton and iron) ${ }^{2}$. We also observed that for some model, the central predicted variance of $\ln \mathrm{A}$ is negative, but this is not the case within our systematic uncertainties.

\section{Hadronic Interactions}

We have performed several analyses to extract a muon size parameter from the hybrid or SD data sets. These analyses [20-23] all indicate that current hadronic interaction models predict muon sizes that are smaller (by at least $20 \%$ ) than those observed in the data, unless one assumes that the data are composed of pure iron which is in contradiction, according to the same models, with the observed $X_{\max }$ distributions.

In Farrar [23], we have selected all showers (411) measured in hybrid mode with an energy between $10^{0.8}$ and $10^{1.2} \mathrm{EeV}$. For each of those showers, we have generated Monte Carlo events with similar energies by selecting those which also matched the measured longitudinal profile. Then, for those matching events, the predicted lateral distribution of the signal has been compared to the data recorded by the SD.

The Monte Carlo predictions have been found to be systematically below the observed signals, regardless of the hadronic model being used. To match the lateral distributions, we introduce two parameters that have been adjusted to the data. These parameters are $R_{E}$ which acts as a rescaling of the shower energy and $R_{\mu}$ which acts as a muon size rescaling factor. The values that best reproduce the data are shown in Fig. 7 for a set of proton showers only and for a

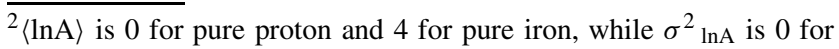
pure composition and 4 for a 50:50 p/Fe mix.
} 


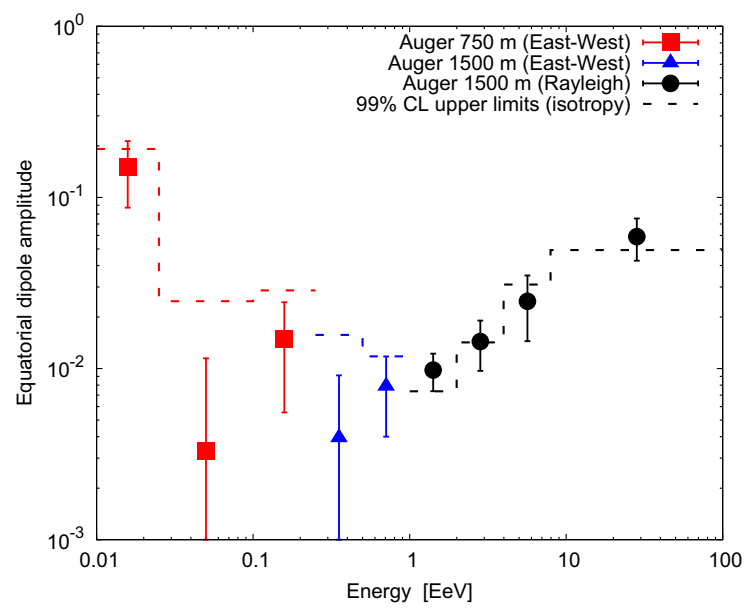

Fig. 9 Equatorial dipole amplitude (left) and phase (right) evolution as a function of energy. Black circle indicates modified Rayleigh analysis, blue triangles East-West analysis, and red squares infill data with

set of showers from a mixed composition of samples whose global $X_{\max }$ distribution matches that of the data.

In all cases, the $R_{\mu}$ rescaling factor is larger than 1 , indicating a deficit in the predictions, while for $R_{E}$, it is compatible with 1 for the mixed set and also for the pure proton set, but only within the systematic uncertainties (mainly originating from our absolute energy scale). Independent analyses using inclined showers or relying on the distinct signal shape left by muons in the WCD also point to a deficit of muons in the simulations [21,22].

In another study, based purely on the SD data, we have reconstructed the muon production depth profile (MPD, [20]). From this profile, it is possible to extract the depth of maximum production of the muons that reach the ground

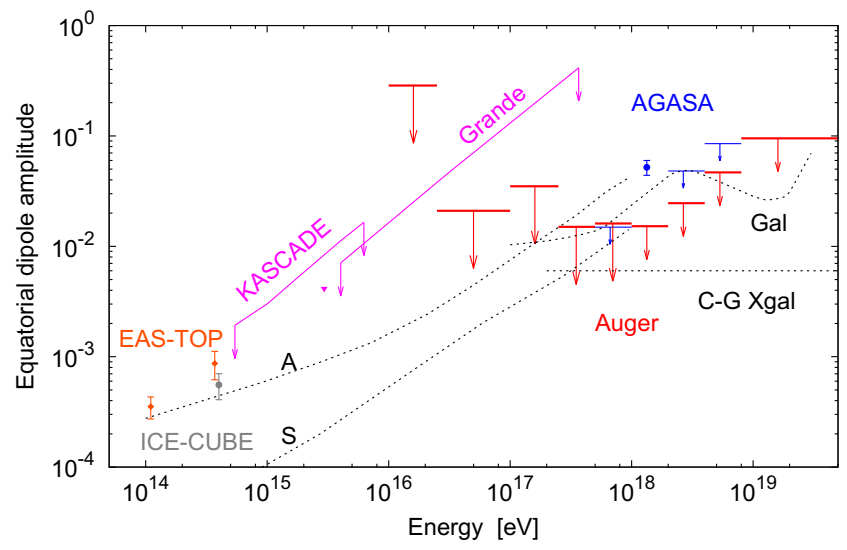

Fig. 10 Upper limit at $99 \%$ CL for the equatorial dipole amplitude as a function of energy. In red are the limits obtained over the full energy range of the Auger Observatory. Results from AGASA are shown in blue, from KASCADE and KASCADE-Grande in magenta, EAS-TOP in orange, and ICE-CUBE in gray. Predictions from different models are displayed, labeled as A, S, Gal, and C-G Xgal (see text) [24]

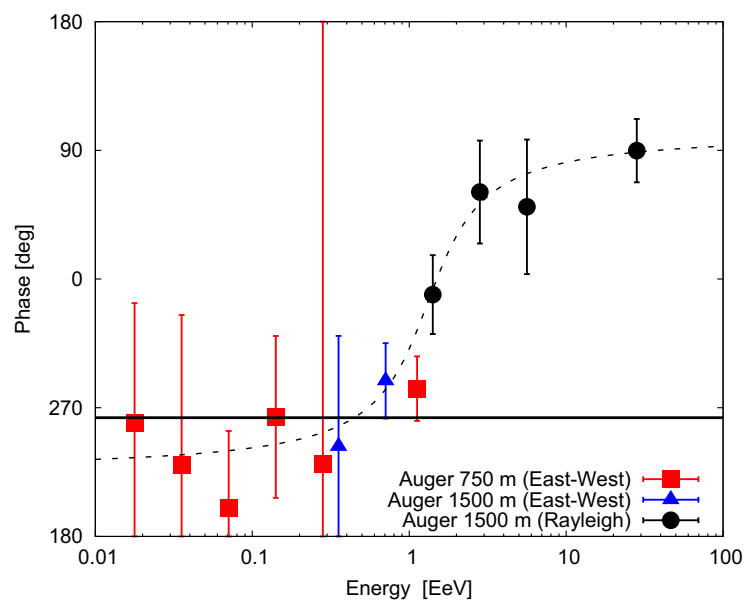

East-West analysis. Three points lie above the $99 \%$ CL line in the amplitude plot, while the phase shows a smooth evolution from the galactic center towards the galactic anticenter directions [24]

$\left(X_{\max }^{\mu}\right)$ which is also a mass indicator as it is linked to the longitudinal evolution of the EAS in the atmosphere.

An interesting aspect of this study is that it gives us a second observable, similar to $X_{\max }$, that can be converted into $\langle\ln \mathrm{A}\rangle$. It is, therefore, tempting to convert both our $X_{\max }$ and $X_{\max }^{\mu}$ data into $\langle\ln \mathrm{A}\rangle$ using the same interaction model. The result of such conversion is shown in Fig. 8 for two models. In the first case, with EPOS-LHC, the two observables convert into an incompatible mass value. According to the model authors [53], this is linked to the better representation of the rapidity gap distribution of $\mathrm{p}$ $\mathrm{p}$ interactions measured at the LHC. Of course, UHECR collision in atmosphere are not p-p collisions, but at least, $\mathrm{p}$-air collisions if not higher masses. The observed apparent contradiction could then simply point at collective effects of the nuclei collisions in the atmosphere. The representation from the second model, QGSJetII-04, seems better, but in that case, the rapidity gap distribution from the

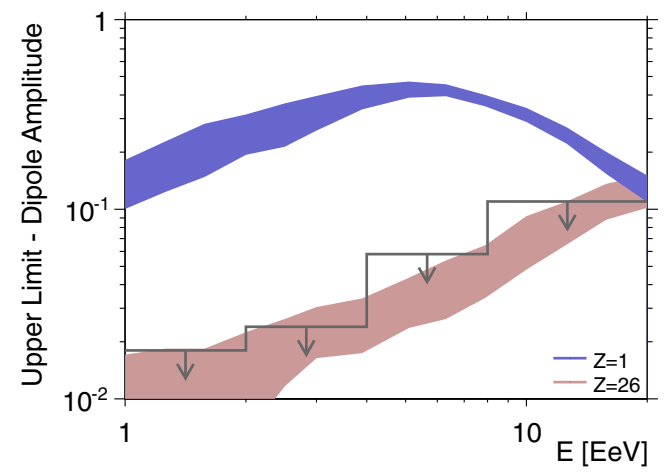

Fig. 11 Upper limit at $99 \%$ CL for the dipole amplitude as a function of energy. Some generic anisotropy expectations from stationary galactic sources distributed in the disk are also shown, for various assumptions on the cosmic ray composition [25] 

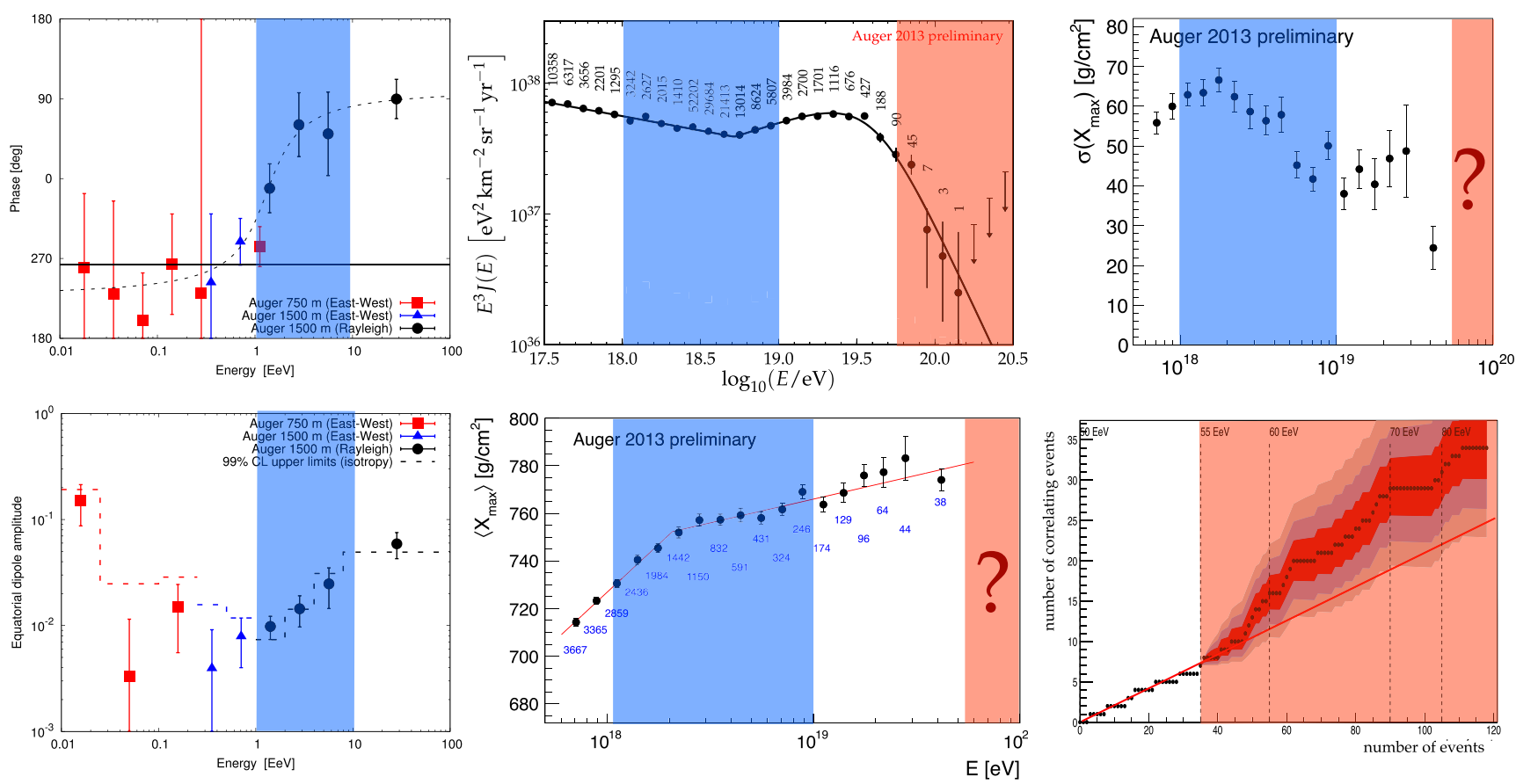

Fig. 12 An overall view of the Auger results showing the variety of the observables and the coherence of their behavior. The blue bands correspond to the ankle region where features are observed in the spectrum, mass, and anisotropy data. The red bands corresponds to the cutoff region where, unfortunately, due to the low duty cycle

model is in poorer agreement with the LHC data. While one cannot conclude on the quality of a given model from this plot alone, this analysis shows the interest and the power of UHECR data to constrain high-energy interaction models.

\section{Anisotropies}

The Auger Collaboration has also performed extended analyses of the UHECR arrival direction distributions in several energy ranges and different angular scales [24-27].

Some particularly interesting results come out of the analysis of the first harmonic modulation in the right ascension distribution of the events [24]. The results of this analysis on the equatorial dipole amplitudes is shown in Fig. 9 for an extended range in energy covering nearly 4 orders of magnitude. While no clear evidence for anisotropy has been found yet, it is remarkable to see that in the range above $1 \mathrm{EeV}$, three out of the four points are above the $99 \%$ CL line, i.e., only $1 \%$ of isotropic samples would show equal or larger amplitudes.

The phase evolution in the same energy range, also shown in Fig. 9, has an interesting behavior with a smooth transition from $270^{\circ}$ (the galactic center direction) to $90^{\circ}$. To test the hypothesis that the phase is undergoing a smooth of the fluorescence technique, the mass information is missing. For completeness, the VCV correlation (from [55]) is also shown as an energy-ordered plot. The onset of the correlation signal is visible at about $55 \mathrm{EeV}$

transition, we began to independently analyze data obtained after April 2011. After 18 months, the new and independent data set is showing a similar trend [24]. Another 18 months of data collection to reach an aperture of $21,000 \mathrm{~km}^{2} / \mathrm{sr}$ with the independent data set is needed before the trend can be confirmed.

It is interesting to note that despite the possible hints for CR anisotropy discussed above, any such anisotropy would be remarkably small (at the percent level). The Auger Collaboration is, therefore, able to place stringent limits on the equatorial dipole amplitude $d_{\perp}$ as shown in Fig. 10. In this figure, the predictions labeled $A$ and $S$ correspond to models in which cosmic rays at $1 \mathrm{EeV}$ are predominant of galactic origin. They escape from the galaxy by diffusion and drift motion, and this causes the predicted anisotropies. A and $S$ stand for two different galactic magnetic field symmetries (antisymmetric and symmetric). In the model labeled Gal [54], a purely galactic origin is assumed for all cosmic rays up to the highest energy. In this case, the anisotropy is caused by purely diffusive motion due to the turbulent component of the magnetic field. Some of these amplitudes are challenged by our current bounds. The prediction labeled C$\mathrm{G} \mathrm{Xgal}$ is the expectation from the Compton-Getting effect for extragalactic cosmic rays due to the motion of our galaxy with respect to the frame of extragalactic isotropy, assumed to be determined by the cosmic microwave background. 
The bounds reported here already exclude the particular model with an antisymmetric halo magnetic field $(A)$ above energies of $0.25 \mathrm{EeV}$ and the Gal model at few $\mathrm{EeV}$ energies and are starting to become sensitive to the predictions of the model with a symmetric field. (see [24] and references therein for more details).

We have also conducted searches for dipole and quadrupole modulations reconstructed simultaneously in declination and right ascension. The upper limits presented in Almeida [25] are shown in Fig. 11. They are presented along with generic estimates of the dipole amplitudes expected from stationary galactic sources distributed in the disk considering two extreme cases of single primaries: protons and iron nuclei. This figure illustrates the potential power of these observational limits.

While other magnetic field models, source distributions, and emission assumptions must be considered, in this particular examples, we can exclude the hypothesis that the light component of cosmic rays comes from stationary sources densely distributed in the galactic disk and emitting in all directions.

\section{Conclusions}

The Auger Observatory is producing measurements of the UHECR properties over 4 orders of magnitude in energy (from $0.01 \mathrm{EeV}$ to above $100 \mathrm{EeV}$ ). A synthesis of those measurements is presented in Fig. 12 where one can scrutinize the quality and coherence of those observations.

The astrophysical interpretation of that data is, however, still delicate as most properties of the UHECR sources are still unknown. When treating the source distributions and cosmological evolutions and their spectral indexes, compositions, and maximum energies as free parameters, many different interpretations can lead to an acceptable reproduction of our $X_{\max }$ spectrum data, leaving alone the fact that all sources need not to be equal! Additionally, the inclusion of our anisotropy results adds more complexity, but there again, the unknowns on the galactic and extragalactic magnetic fields and on the source distributions and composition leave much space for speculations.

Nevertheless, taking at face value the current model conversion of our $X_{\max }$ data into masses and adding the information of our spectrum measurement, it is possible that the cutoff region represents more of a consequence of the source maximal acceleration energy (of the order of $4 \mathrm{EeV}$ for proton) than a propagation effect as expected from the GZK scenario. However, taking into account the remaining nontrivial correlation observed in our highest energy events with the VCV catalog (see Fig. 12, the correlation signal is $2 \sigma$ above the expected fraction for an isotropic sky), the presence of a subdominant fraction (less than about $20 \%$ ) of protons may be expected in this region. The identification of this subdominant fraction will require an excellent mass determination capability in this energy range-something similar to the current FD performances on the measurement of the EAS longitudinal development, but with a $100 \%$ duty cycle. Note also that in such scenarios, the spectral features originate from the source properties rather than from an interaction of the bulk of the cosmic rays with the CMB. Magnetic deflections in transit to the Earth are also important.

Still in the cutoff region, another interpretative option is to consider a possible change in the hadronic interactions of protons at the highest energy. Such modification would make the proton EAS look like those currently modeled from heavier nuclei. The difficulty encountered in constraining the high-energy interaction generators at energies 1 or 2 orders of magnitude above the LHC leaves some room for such a scenario. Additional data from UHECR including, in particular, the muonic content of EAS will definitely help in reducing those unknowns.

In the ankle region, the question is still open as to whether the break observed in the spectrum is the consequence of a propagation effect or the signature of a transition between two types of sources (be they both galactic or not). Several key observables, if they are combined, will help to resolve the issue. An anisotropy study for at least two different mass spectra (one light and one heavy) from $0.1 \mathrm{EeV}$ up to $10 \mathrm{EeV}$ would, for example, allow to distinguish between a propagation effect and a source transition scenario. The key is to cover a wide enough energy range to connect adequately the new data to that measured by observatories at lower energies such as those from KASCADE-Grande [56].

Additional information such as the limits on the photon fractions in the $\mathrm{EeV}$ range and/or the neutrino fluxes will also bring interesting light into both regions. The absence of cosmogenic photons or neutrinos, for example, would indicate clearly that there are no (or very few) proton sources in the cosmos with limiting energy well above the GZK cutoff.

The Auger Observatory will continue taking data for the years to come, and the collaboration is deeply engaged in improvements and upgrades of our detection systems. We aim at covering the open issues discussed above.

At the low-energy end (between 0.01 and $1 \mathrm{EeV}$ ), we have the HEAT and AMIGA extensions. We have also recently modified the local trigger conditions of the surface array detectors to lower our full trigger efficiency threshold. It is now about $1 \mathrm{EeV}$ for the $1.5 \mathrm{~km}$ array (it was $3 \mathrm{EeV}$ before). This improvement will provide us with about five times more events in this energy range than what we had before. This will allow us to augment significantly our sensitivity to anisotropy searches. In addition, because this new triggering scheme is less sensitive to individual muons entering the WCDs, it will allow us to improve significantly 
our photon sensitivity. Together with the increased statistics, this opens great perspectives for the cosmogenic photon searches.

At the high-energy end, the upgrade of our SD array is understudied to provide us with a detector able to measure both the muon content and the age of the shower at ground. This two observables will give us the means to identify the UHECR composition on an event-by-event basis up to the highest energy. The collaboration is evaluating several detector options that can, in principle, fulfill these ambitious scientific goals [57].

Acknowledgments The successful installation, commissioning, and operation of the Pierre Auger Observatory would not have been possible without the technical and administrative staff in Malarguie. We acknowledge their extraordinary commitment to this scientific endeavor. ALS acknowledges financial supports by the French National Institute for nuclear and particle physics (CNRS/IN2P3) and by the University Pierre \& Marie Curie in Paris (UPMC).

\section{References}

1. The Pierre Auger Collaboration, NIM A. 523, 50-95 (2004)

2. The Pierre Auger Collaboration, NIM A. 613, 29-39 (2010)

3. The Pierre Auger Collaboration, NIM A. 620, 227-251 (2010)

4. F. Sánchez for the Pierre Auger coll., 32nd ICRC proceedings, arXiv: 1107.4807

5. T. Hermann-Josef Mathes for the Pierre Auger coll., 32nd ICRC proceedings, arXiv: 1107.4807

6. J.L. Kelley for the Pierre Auger coll., 32nd ICRC proceedings, arXiv: 1107.4807

7. R. Gaïor for the Pierre Auger Coll., these proceedings, arXiv:1307.5059; and R. Gaïor, PhD thesis, University of Paris 6 (UPMC), September 2013

8. P. Allison for the Pierre Auger coll., 32nd ICRC proceedings, arXiv:1107.4807

9. J. Alvarez-Muñiz et al., arXiv: 1208.2734

10. The Pierre Auger Collaboration, arXiv: 1307.5059 contains all the Auger contributions to the 33rd ICRC listed below (all authors to be understood "for the Pierre Auger Collaboration")

11. V. Verzi, The energy scale of the Pierre Auger Observatory. CREX 928

12. M.J. Tueros, Estimate of the non-calorimetric energy of showers observed with the fluorescence and surface detectors of the Pierre Auger Observatory. CR-EX 705

13. J. Bäuml, Measurement of the optical properties of the auger fluorescence telescopes. CR-IN 806

14. C. Bonifazi, The monitoring system of the Pierre Auger Observatory: on-line and long- term data quality controls. CR-IN 1079

15. D. Veberic, Estimation of signal in saturated stations of Pierre Auger surface detector. CR-IN 633

16. A. Schulz, Measurement of the energy spectrum of cosmic rays above $3 \times 10^{17} \mathrm{eV}$ with the Pierre Auger Observatory. CR-EX 769

17. D. Ravignani, Measurement of the energy spectrum of cosmic rays above $3 \times 10^{17} \mathrm{eV}$ using the AMIGA $750 \mathrm{~m}$ Surface Detector Array of the Pierre Auger Observatory. CR-EX 693

18. V. de Souza, An update on the measurements of the depth of shower maximum made at the Pierre Auger Observatory. CR-EX 751
19. E.-J. Ahn, Inferences about the mass composition of cosmic rays from data on the depth of maximum at the Auger Observatory. CR-EX 690

20. D. García-Gámez, Observations of the longitudinal development of extensive air showers with the surface detectors of the Pierre Auger Observatory. CR-EX 694

21. I. Valiño, A measurement of the muon number in showers using inclined events recorded at the Pierre Auger Observatory. CR-EX 635

22. B. Kégl, Measurement of the muon signal using the temporal and spectral structure of the signals in surface detectors of the Pierre Auger Observatory. CR-EX 860

23. G.R. Farrar, The muon content of hybrid events recorded at the Pierre Auger Observatory. CR-EX-1108

24. I. Sidelnik, Measurement of the first harmonic modulation in the right ascension distribution of cosmic rays detected at the Pierre Auger Observatory: towards the detection of dipolar anisotropies over a wide energy range. CR-EX 739

25. R.M. de Almeida, Constraints on the origin of cosmic rays from large scale anisotropy searches in data of the Pierre Auger Observatory. CR-EX 768

26. B. Revenu, Blind searches for localized cosmic ray excesses in the field of view of the Pierre Auger Observatory. CR-EX 1206

27. F. S. Greus, Searches for galactic neutron sources with the Pierre Auger Observatory. CR-EX 1125

28. D. Kuempel, Directional search for ultra-high energy photons with the Pierre Auger Observatory. CR-EX 669

29. P. Pieroni, Ultra-high energy neutrinos at the Pierre Auger Observatory. CR-EX 697

30. F. Suarez, The AMIGA muon detectors of the Pierre Auger Observatory: overview and status. CR-IN 712

31. S. Maldera, Measuring the accuracy of the AMIGA muon counters at the Pierre Auger Observatory. CR-IN 748

32. F.G. Schröder, Radio detection of air showers with the Auger Engineering Radio Array. CR-IN 899

33. T. Huege, Probing the radio emission from cosmic-ray-induced air showers by polarization measurements. CR-EX 661

34. R. Gaïor, Detection of cosmic rays using microwave radiation at the Pierre Auger Observatory. CR-IN 883

35. L. Valore, Measuring atmospheric aerosol attenuation at the Pierre Auger Observatory. CR-EX 920

36. M.I. Micheletti, Aerosol characterization at the Pierre Auger Observatory. CR-EX 1081

37. J. Chirinos, Cloud monitoring at the Pierre Auger Observatory. CR-EX 994

38. A. Tonachini, Observation of Elves at the Pierre Auger Observatory. CR-EX 676

39. G.R. Snow, Education and outreach activities of the Pierre Auger Observatory. CR-IN 968

40. Telescope array and Pierre Auger Collaboration, Pierre Auger Observatory and Telescope Array: Joint Contributions to the 33rd International Cosmic Ray Conference (ICRC 2013), arXiv:1310.0647 [astro-ph.HE]

41. J.N. Matthews, for the Pierre Auger and Telescope Array coll., Progress Towards a Cross-Calibration of the Auger and Telescope Array Fluorescence Telescopes via an Air-borne Light Source. CR-IN 1218, these proceedings

42. O. Deligny for the Pierre Auger and Telescope Array coll., Measuring Large-Scale Anisotropy in the Arrival Directions of Cosmic Rays Detected at the Telescope Array and the Pierre Auger Observatory above $10^{19} \mathrm{eV}$. CR-EX 679, these proceedings

43. W.F. Hanlon, for the Pierre Auger and Telescope Array coll., Progress towards understanding the analyses of mass composition made by the Auger and Telescope Array Collaborations. CR-EX 964 , these proceedings 
44. H. Tokuno, et al. for the Telescope Array Coll., NIM. A676, 5465 (2012)

45. A. Letessier-Selvon, T. Stanev, Rev. Mod. Phys. 83, 907 (2011)

46. M. Ave, et al., Astropart. Phys. 42, 90 (2013)

47. K.-H. Kampert, et al., Astropart. Phys. 42, 41 (2013)

48. R. Aloisio, et al., JCAP10, 007 (2012)

49. E.J. Ahn, et al., Phys. Rev. D. 80, 094003 (2009)

50. T. Pierog, et al. arxiv:1306.0121

51. S. Ostapchenko, Phys. Rev. D. 38, 014018 (2011)
52. The Pierre Auger Collaboration, JCAP. 02, 026 (2013)

53. T. Pierog, private communication

54. A. Calvez, A. Kusenko, S. Nagataki, Phys. Rev. Lett. 105, 091101 (2010)

55. K.-H. Kampert for the Pierre Auger Collaboration, Highlights from the Pierre Auger Observatory. arXiv:1207.4823

56. KASCADE-Grande collaboration, Phys. Rev. D. 87, 081101(R) (2013)

57. The Pierre Auger Collaboration, Plans for a proposal to upgrade the Pierre Auger Observatory, in preparation 